\title{
Islets of Langerhans: The Puzzle of Intraislet Interactions and Their Relevance to Diabetes
}

Gordon C. Weir and Susan Bonner-Weir

Joslin Diabetes Center, New England Deaconess Hospital, Brigham and Women's Hospital, and Harvard Medical School, Boston, Massachusetts 02215

The islets of Langerhans, discovered by a medical student 121 years ago (1), occupy a pivotal position in the endocrine control of fuel metabolism. In the past decade, research has provided exciting insights into the remarkable sophistication of these microorgans. The purpose of this perspective is to focus upon the complex interactions that take place between islet cells. Communication between adjacent cells of the same type can occur; these interactions have been shown best in pancreatic B cells. Different cell types can interact through paracrine mechanisms, in which a secretory product moves a short distance through interstitial fluid to reach a target cell. Communication can also take place via a local vascular portal system within islets and, in addition, there is coordination of function between islets. Delineation of these complicated relationships has contributed to our understanding of normal physiology and the pathogenesis of diabetes mellitus.

\section{Islet anatomy}

The islets of Langerhans, taken together, can be thought of as a single organ occupying $\sim 1 \%$ of the pancreas. The human pancreas contains over 1 million islets, and these typically consist of about 2,500 cells, although some may be collections of only a few cells and others may be made up of over 12,000 cells. There is an understandable advantage in having the islet organ placed so that insulin and glucagon are directly secreted into the portal vein, thereby optimizing their control over hepatic function. The potential advantage of having islets diffusely distributed instead of being merged into a single solid organ is more mysterious. One suggestion is that there is a local islet-acinar portal vascular system that could allow exocrine tissue to be nourished by islet hormones (2); this may have been important during some stages of evolution.

An appreciation of the anatomy of islets is crucial for understanding the function of these complex microorgans. Islets have a central core of insulin-containing B cells and a surrounding mantle of glucagon-containing A cells, somatostatin containing D cells, and pancreatic polypeptide-containing PP cells (see cover figure). In some mammalian species, such as in horse and even man, the pattern may not be as well defined as in the rat, but the B cells are always clumped together with an

Address reprint requests to Dr. Weir, Joslin Diabetes Center, 1 Joslin Place, Boston, MA 02215.

Received for publication 16 January 1990.

J. Clin. Invest.

(c) The American Society for Clinical Investigation, Inc.

0021-9738/90/04/0983/05 \$2.00

Volume 85, April 1990, 983-987 adjacent mix of non-B cells. When rat islets are dispersed into single cells and allowed to reaggregate in tissue culture, the B cells form a central core with non-B cells attaching to the outside, re-establishing the native pattern (3).

The largest portion of the pancreas, the dorsal lobe, is derived from the embryonic dorsal anlage, but a smaller portion, the ventral lobe making up the inferior aspect of the head, is derived from the ventral anlage. The islets of the dorsal lobe have mantles which contain mostly A cells and D cells, whereas the islets of the ventral lobe have mantles that consist mostly of PP and D cells. Islets are innervated by sympathetic nerves, parasympathetic nerves, and the poorly understood peptidergic nerves (4). Species variation in the distribution of islet neurons is considerable which is consistent with observed differences in autonomic control.

Islets are richly vascularized by direct arteriolar blood flow. Studies with microspheres have shown that islets, which make up only $1 \%$ of the mass of the pancreas, receive at least $10 \%$ of the blood flow (5). Small arterioles enter the B cell-containing core of the islet through discontinuities or pores of the mantle, and then break into a glomerular-like network of fenestrated capillaries which coalesce into collecting venules either during or after leaving the islet (6). As will be discussed in more detail, this arrangement creates a portal system in which insulin can be carried in high concentrations to the mantle cells.

The microvasculature of the core has a unique relationship with B cells, which are now known to be polarized with one domain facing an arterial capillary and another facing a venous capillary (7). The B cells resemble other epithelial cells, having a basolateral portion and an apical portion; the latter contains relatively more insulin secretory granules. In addition, as shown in Fig. 1, there are canaliculi, which are located between the lateral surfaces of $B$ cells, and span the distance from the arterial to the venous domains (8). These canaliculi contain large numbers of microvilli, which have been found to be enriched in the recently described $B$ cell glucose transporter (9). The presence of these elaborate structures suggests that interstitial fluid flows through these canaliculi in an arterial to venous direction and that glucose uptake of B cells is carried out primarily by the transporters on these microvilli. Thus, these canaliculi may serve as the initial interface for glucose sensing by the B cell. Through these glucose transporters extracellular glucose rapidly equilibrates with cytosolic glucose and is phosphorylated, with glucokinase serving a key rate-limiting function; glucose metabolism is tightly linked to the rate of insulin secretion (10). Insulin secretory granules appear to be released from the lateral and apical surfaces to enter the venous capillaries.

Less is known about polarization of the mantle cells and their relationship to blood vessels, although A cells have been 


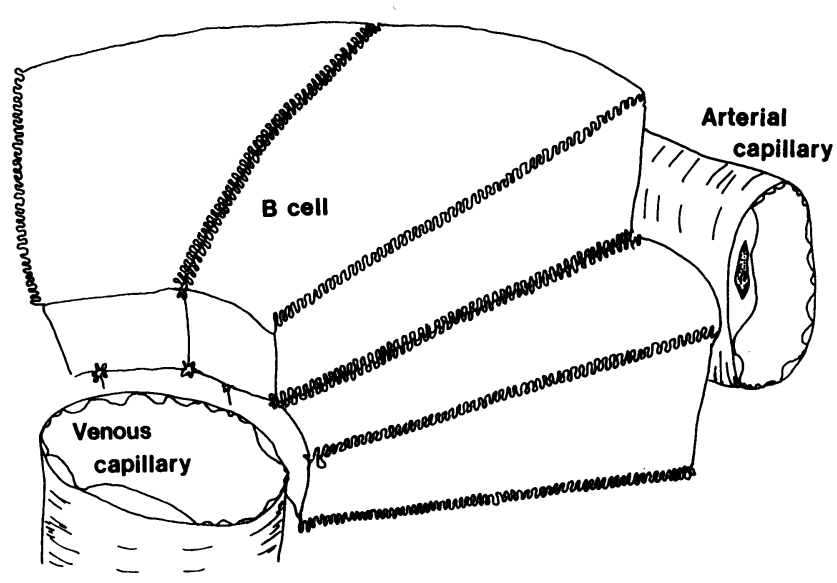

Figure 1. Diagram of the intraislet arrangement of pancreatic B cells. $B$ cells have two capillary faces, one arterial and the other venous. The lateral interfaces of B cells are smooth surfaces. However, where three or more B cells meet, a canalicular system is found that extends from one capillary face to the other. The canaliculi contain microvilli that are enriched in glucose transporters. This specialized arrangement suggests that interstitial fluid may flow through these canaliculi in an arterial to venous direction.

found in a columnar cordlike arrangement with the majority of cells facing two separate blood vessels (11). Mantle cells presumably are exposed to very high concentrations of bloodborne insulin, but virtually nothing is known about whether separate sensing or secretory domains are present. Islet $D$ cells have been described as having a dendritic shape and can penetrate into the outer aspect of the B cell core (12). The projections of these cells contain granules and this could be an anatomical basis for possible paracrine effects of $D$ cells upon B cells. Granule-containing portions of $D$ cells are also adjacent to A and PP cells. Because somatostatin has such potent inhibitory effects upon insulin, glucagon, and pancreatic polypeptide secretion, it will be of considerable interest to learn more about the anatomical relationships between $D$ cells and the other islet cell types.

\section{Insulin secretion}

Although B cells are usually thought of as being identical and of functioning in a synchronous manner, evidence of heterogeneity of both structure and function is accumulating. For example, B cells in the center of the islet core are more degranulated after a glucose challenge than B cells in the periphery of the core (13). Also, there are several studies indicating that the thresholds for glucose stimulation of insulin secretion and biosynthesis vary considerably among $B$ cells $(14,15)$. There is even evidence that stimulated $B$ cells of the dorsal lobe release more insulin than those in the ventral lobe. Virtually nothing is known about the mechanisms that underlie this heterogeneity. Presumably some of the differences result from variations in the local environment and some are intrinsic to the cells. Important questions are raised, however, such as whether certain populations of B cells might be more susceptible to autoimmune destruction or whether there might be variability in $B$ cell performance and survival during the development of non-insulin-dependent diabetes mellitus (NIDDM). ${ }^{1}$

1. Abbreviations used in this paper: IDDM, insulin-dependent diabetes mellitus; NIDDM, non-insulin-dependent diabetes mellitus.
The question of how B cells communicate between one another has received considerable attention. Electrophysiological studies have shown that the electrical activity of B cells within a given islet is synchronous. In addition, insulin secretion from a single islet has been shown to correlate with electrical activity (16), even though there could still be considerable variability of secretion among the $2,500 \mathrm{~B}$ cells contained in such an islet. Indeed, studies with isolated B cells indicate that B cells that do not secrete insulin in response to glucose still maintain characteristic electrical responses (17). Gap junctions are found between B cells, as well as between B cells and non-B cells, and these can permit passage of electric current and small molecules of $\angle 900 \mathrm{D}$ between cells (18). These may be the pathways that coordinate electrical communication between $B$ cells, thus being important for the integrity of insulin secretion. The role of gap junctions in non-B cells is even less well understood.

Not only do B cells communicate within islets, but there is also communication between islets. Oscillation of insulin and glucagon concentrations in plasma has been found with the periodicity being between 12 and $15 \mathrm{~min}$ in normal man (19). The oscillation patterns can be maintained by the pancreas alone as has been shown in studies utilizing the isolated perfused canine pancreas (20). The mechanisms through which islets synchronize their activity are not understood, but there is probably some kind of intrinsic neural coordination that persists even after nerves that supply the pancreas are severed. The oscillations are lost in NIDDM, a finding of uncertain significance (21). There have been suggestions that the oscillations provide greater efficiency of insulin and glucagon action upon the liver.

\section{Glucagon secretion}

Glucagon has an important influence upon glucose and ketone metabolism, and its secretion is strongly influenced by changes in plasma glucose concentration. Normally, an increase in glucose level suppresses glucagon secretion while simultaneously stimulating insulin secretion. A critical question is: How much of the glucose effect is exerted directly upon the pancreatic A cell and how much is done indirectly through other islet cells and the autonomic nervous system? It has been difficult to obtain an answer to the first question because in the ideal experiment the effects of $B$ and $D$ cells and the autonomic nervous system must be controlled. In rat A cells isolated with cell sorting techniques, glucagon secretion could be suppressed by glucose (22). The mechanisms of this action by glucose are unknown, but glucose appears to depress cytosolic calcium levels in rat A cells (23). These are important first steps, but other approaches must be devised to provide a more complete assessment.

The indirect effects of glucose upon glucagon secretion have been studied in many ways, and there is a strong circumstantial link between B and A cell responses. Whenever glucose-induced insulin secretion is lost, as is found in human and many types of experimental diabetes, glucose can no longer suppress glucagon release $(24,25)$. Much of A cell suppression by glucose is thought to be dependent upon the high concentrations of insulin, which are carried by the local portal vascular system from the B cell-rich core to the islet mantle (6). This portal system has been comprehensively studied by Samols and Stagner with passive immunization using antisera against insulin, glucagon, and somatostatin, infused into iso- 
lated pancreases in both antegrade and retrograde directions (26). These experiments have been carried out in the pancreases of rats, dogs, and primates; in all of these systems the interactions occur in a downstream direction, from $B$ to $A$ to $D$ cells. Thus, in normal islets, $A$ and $D$ cells are influenced by insulin carried by the portal system, but B cells are not vascularly influenced by glucagon or somatostatin. Also, A cells are not affected by somatostatin in this local vascular compartment. These experiments only shed light on the interactions that are mediated through the local islet vasculature and do not exclude paracrine interactions.

Paracrine effects are far more difficult to evaluate because of the lack of good methods for studying the diffusion of peptides throughout interstitial compartments. Presumably the diffusion of these islet peptides is limited by the direction of the flow of interstitial fluid and by anatomical barriers. For instance, polarized cells may only respond to peptides that have access to a particular "sensing" face. Some compartmentalization is present because the arterial delivery of very small concentrations of somatostatin, far lower than must be present in some portions of islets, can inhibit glucagon secretion. Therefore, at least some domains of A cells must be protected from local somatostatin secretion. Workers in the field eagerly await the development of specific insulin, glucagon, and somatostatin receptor antagonists which can easily penetrate into interstitial spaces. This will make it possible to study the influences of endogenous islet hormones in intact tissue. An important caveat about studying intraislet interactions is that the anatomical integrity of the islet must always be a foremost consideration. Normal vascular, neural, and interstitial relationships are disrupted in such systems as isolated islets, islet monolayer cultures, and isolated cell models; therefore, results obtained in such systems may not be physiologically relevant.

Pancreatic A cells could be suppressed by glucose through indirect pathways other than intraislet insulin; the candidate mediators being $\mathrm{D}$ cells and/or the autonomic nervous system. For example, high glucose concentrations can stimulate the release of somatostatin (27), a very potent inhibitor of glucagon secretion. A and D cells are anatomically adjacent and therefore paracrine suppression of glucagon by this indirect pathway remains an attractive hypothetical possibility; of course, local glucagon influence over the D cell could also occur (Fig. 2). Of note, glucose stimulation of somatostatin prevails over other theoretical influences upon the pancreatic D cell, these being the potential inhibitory effects of local insulin secretion and decreased paracrine glucagon release. This web of possibilities highlights the potential complexity of islet interactions; surely coordination between dominating and modulating influences exists.

Changes in plasma glucose concentration are associated with changes in autonomic nervous system activity that might be expected to influence islet secretion, as occurs during severe stress. Hypoglycemia is associated with activation of the parasympathetic nervous system and release of epinephrine from the adrenal medulla, but with little change in the release of norepinephrine from the other branch of the sympathetic nervous system (28). Although the autonomic nervous system may have an important role in stimulating glucagon secretion during hypoglycemia in some species, extensive studies indicate that this mechanism contributes little if anything to the increases of glucagon in man (29). An interesting study in an isolated perfused rat pancreas system found that the glucagon

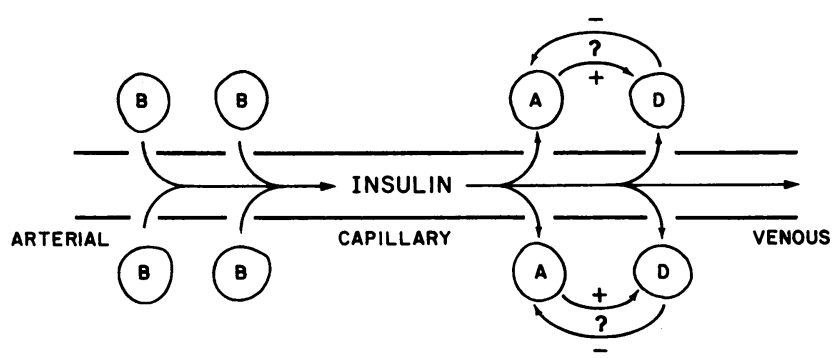

Figure 2. A schematic representation of the anatomical basis for interactions between pancreatic islet cells. Insulin secreted by B cells in the islet core can reach the A and D cells of the mantle via the portal vasculature. In addition, D cells can be influenced by glucagon secreted by A cells into this same vascular compartment. The potential paracrine (nonvascularly mediated) interactions that may occur between adjacent $A$ and $D$ cells have not yet been elucidated.

rise following a reduction in glucose concentration could be attenuated by the alpha adrenergic blockade (30). This finding suggests that hypoglycemia can directly activate the sympathetic nervous system in specific organs, independently of the central nervous system. However, the finding that phentolamine has no influence upon the glucagon increase seen during insulin-induced hypoglycemia in man suggests that this unique hypothetical mechanism is not important in the human pancreas.

\section{Somatostatin and pancreatic polypeptide}

Somatostatin has potent inhibitory effects upon insulin, glucagon, and pancreatic polypeptide secretion, but its role within the islet continues to be a mystery. Certainly islet somatostatin has no endocrine influence because the amount secreted is dwarfed by the amount released from the intestine. The predominant form of somatostatin secreted by the pancreatic $D$ cell is the tetradecapeptide somatostatin-14, rather than the $\mathrm{NH}_{2}$-terminally extended form somatostatin-28, which is found in intestine (31). Somatostatin-14 secreted by islet D cells is generally assumed to have paracrine effects upon $A$ and PP cells and perhaps upon B cells at the periphery of the islet core. Aside from the vague suggestion that it is part of a complex, redundant control system, there are few attractive hypotheses about how somatostatin might be important in islets. Perhaps somatostatin has a more important function in nonmammalian species. Unfortunately, the experimental approaches that have been used to date have not advanced our understanding of islet somatostatin's role in either normal physiology or diabetes. Even less is known about the PP cell and pancreatic polypeptide, but this peptide's inhibitory effect on pancreatic exocrine secretion suggests a potential physiological role (32).

\section{Islet cell interactions in diabetes}

Islet secretion is drastically altered in virtually all forms of diabetes. There are quantitative changes, the most obvious one the reduced insulin secretion that accompanies B cell deficiency. In addition, there are qualitative changes in which normal control of secretion becomes deranged. Insulin secretion has been extensively characterized in NIDDM and to a lesser degree in insulin-dependent diabetes mellitus (IDDM); in both there is profound unresponsiveness of B cells to changes in glucose concentration with some preservation of 
responses to various nonglucose secretagogues. A leading hypothesis supported by studies in experimental animals, suggests that these changes in B cell function result from the deleterious effects of chronic hyperglycemia (33). Altered A cell responses to glucose in diabetes have also been extensively characterized, and the pancreatic D cell has been found to be unresponsive to glucose in an experimental animal model (34).

In IDDM, plasma glucagon concentrations are elevated in either an absolute or relative sense, as hyperglycemia would normally be expected to suppress A cell secretion (24). This issue is of interest because glucagon contributes to the hyperglycemia of IDDM, although it must be stated that the practical importance of this is uncertain. The most compelling hypothesis about the mechanism responsible for this abnormality, supported by experiments in man and experimental animals, is that deficient intraislet insulin secretion is at fault $(23,24)$. The dependence of A cell responsiveness to glucose upon intraislet insulin is complex. In the nondiabetic state, A cells appear to be influenced by enormous fluctuations in the concentration of intraislet insulin concentration, which hypothetically should exceed $20,000 \mu \mathrm{U} / \mathrm{ml}$ during glucose stimulation (35). Little influence is exerted by the lower insulin concentrations that circulate in peripheral plasma (36). In contrast, A cell sensitivity to insulin in IDDM appears to have changed, perhaps through changes in insulin receptor number, among other potential mechanisms, and suppression can be exerted by levels of insulin which are even lower than 100 $\mu \mathrm{U} / \mathrm{ml}$ (37). This suggests that in insulin-treated IDDM, exogenously administered insulin exerts a tonic inhibition of glucagon release, even though plasma glucagon levels remain inappropriately elevated.

Mechanisms other than intraislet insulin could be contributing to the lack of suppressibility of glucagon secretion by glucose in IDDM. For example, chronic hyperglycemia could have a direct deleterious influence upon the A cell that could render it insensitive to glucose change, as was suggested before. Unfortunately, because of the difficulty of designing experiments which adequately control the influence of either endogenous or exogenous insulin, it has been very difficult to test this hypothesis. In addition, any normal or abnormal change in glucagon secretion could be related to somatostatin, but such a possibility remains only hypothetical.

The failure of glucagon concentrations to rise during insulin-induced hypoglycemia in IDDM is a matter of concern, particularly in this era of aggressive insulin management, because glucagon, along with epinephrine, provides an important defense against hypoglycemia. The mechanisms responsible for this defect are not well defined, but deficient intraislet insulin is a leading possibility. In the nondiabetic situation, a fall in glucose level is accompanied by a decrease in intraislet insulin, which may in turn lead to increased glucagon secretion; this cannot occur once the B cell population is severely depleted by autoimmune destruction. Glucagon responsiveness to hypoglycemia persists in the early stages of IDDM and its loss can at least partially be correlated with a decline of residual B cell function (38). Defective autonomic function is not an attractive explanation because the A cell unresponsiveness develops long before autonomic neuropathy is found; furthermore, the autonomic nervous system does not seem to be responsible for the hypoglycemia-induced glucagon increases found in nondiabetic humans (29). The possibility that the defect is caused by a direct effect of hyperglycemia upon the A cell also does not fit, because glucagon responses are at least partially intact in the presence of the hyperglycemia found in early IDDM.

Glucagon secretion is also abnormal in NIDDM in which glucagon secretion is not appropriately suppressed by hyperglycemia and the glucagon responses to insulin-induced hypoglycemia are reduced (39). Severe deficiency of intraislet insulin release is not present in NIDDM, but B cells are unresponsive to both increases and decreases in glucose concentration (40). Thus, failure of intraislet insulin to rise during a glucose challenge could lead to an impairment of glucagon suppression and, likewise, a sluggish fall of intraislet insulin during hypoglycemia could also result in an impaired glucagon response. Similar pathophysiological mechanisms appear to take place in experimental animal models of NIDDM (41).

The progress that has been made in working through the puzzle of islet interactions has been impressive. Nonetheless, we still have much to learn about this complex system and how it is altered by such situations as autoimmune destruction, the deterioration of islet function in NIDDM, and the many challenges that will be found in the important field of islet and pancreas transplantation.

\section{Acknowledgments}

We thank Dr. Fannie Smith for preparation of the in situ hybridization shown on the cover of this issue and James Evans for assistance with the manuscript.

This work was supported by the following grants from the National Institutes of Health, DK-35449 and DK-36836 (Diabetes Center grant).

\section{References}

1. Langerhans, P. 1937. Contributions to the microscopic anatomy of the pancreas. Bull. Inst. History Med. 5:1-39.

2. Henderson, J. R. 1969. Why are the islets of Langerhans? Lancet. ii:469-470.

3. Halban, P. A., S. L. Powers, K. L. George, and S. Bonner-Weir. 1987. Spontaneous reassociation of dispersed adult rat pancreatic islet cells into aggregates with 3-dimensional architecture typical of native islets. Diabetes. 36:783-791.

4. Ahren, B., G. J. Taborsky, and D. Porte. 1986. Neuropeptidergic as cholinergic and adrenergic regulation of islet hormone secretion. Diabetologia. 29:827-836.

5. Lifson, N., K. G. Kramlinger, R. R. Mayrand, and E. J. Lender. 1980. Blood flow to the rabbit pancreas with special reference to the islets of Langerhans. Gastroenterology. 79:466-473.

6. Bonner-Weir, S., and L. Orci. 1982. New perspectives on the microvasculature of the islets of Langerhans in the rat. Diabetes. 31:883-339.

7. Bonner-Weir, S. 1988. Morphological evidence for pancreatic polarity of B-cell within the islets of Langerhans. Diabetes. 37:616621 .

8. Bonner-Weir, S. 1989. Potential "sensing" and "secreting" domains of the pancreatic B-cell. Diabetes. 2:98a. (Abstr.)

9. Orci, L., B. Thorens, M. Ravazzola, and H. F. Lodish. Localization of the pancreatic beta cell glucose transporter to specific plasma membrane domains. Science (Wash. DC). 245:295-297.

10. Meglasson, M. D., and F. M. Matschinsky. 1986. Pancreatic islet glucose metabolism and regulation of insulin secretion. Diabetes/ Metab. Rev. 2:163-214. 
11. Bonner-Weir, S. 1989. Pancreatic islets: morphology, organization, and physiological implications. In Insulin Secretion. B. Draznin, S. Melmed, and D. LeRoith, editors. Alan R. Liss, New York. 1-11.

12. Leiter, E. H., D. A. Gapp, J. J. Eppig, and D. L. Coleman. 1979. Ultrastructural and morphometric studies of delta cells in pancreatic islets from C57BL/Ks diabetes mice. Diabetologia. 17:297-309.

13. Stefan, Y., P. Meda, M. Neufeld, and L. Orci. 1987. Stimulation of insulin secretion reveals heterogeneity of pancreatic B-cells in vivo. J. Clin. Invest. 80:175-183.

14. Salomon, D., and P. Meda. 1986. Heterogeneity and contact dependent regulation of hormones secretion by individual B-cells. Exp. Cell Res. 162:507-520.

15. Schuit, F. C., P. A. In'tveld, and D. G. Pipeleers. 1988. Glucose stimulates proinsulin biosynthesis by a dose-dependent recruitment of pancreatic beta cells. Proc. Natl. Acad. Sci. USA. 85:3865-3869.

16. Scott, A. M., I. Atwater, and E. Rojas. 1981. A method for simultaneous measurement of insulin release and B-cell membrane potential in single mouse islets of Langerhans. Diabetologia. 21:470475 .

17. Soria, B., M. Chanson, and P. Meda. 1989. Glucose responsive and unresponsive B-cells show similar electrical characteristics. Diabetologia. 32:543a. (Abstr.)

18. Meda, P., A. Perrelet, and L. Orci. 1984. Gap junctions and cell-to-cell coupling in endocrine glands. Mod. Cell Biol. 3:131-196.

19. Lang, D. A., D. R. Matthews, J. Peta, and R. C. Turner. 1979. Cyclic oscillations of basal plasma glucose and insulin concentrations in human beings. N. Engl. J. Med. 301:1023-1027.

20. Stagner, J., E. Samols, and G. Weir. 1980. Sustained oscillations of insulin, glucagon and somatostatin from the isolated canine pancreas during exposure to a constant glucose concentration. J. Clin. Invest. 65:939-942.

21. O'Rahilly, S., R. C. Turner, and D. R. Matthews. 1988. Impaired pulsative secretion of insulin in relatives of patients with noninsulin-dependent diabetes. N. Engl. J. Med. 318:1225-1230.

22. Pipeleers, D. G. 1987. The biosociology of pancreatic B-cells. Diabetologia. 30:277-291.

23. Wang, J.-L., and M. L. McDaniel. 1990. Secretagogue-induced oscillations of cytoplasmic $\mathrm{Ca}^{2+}$ in single $\beta$ and $\alpha$ cells obtained from pancreatic islets by fluorescence-activated cell sorting. Biochem. Biophys. Res. Commun. In press.

24. Unger, R. H. 1985. Glucagon physiology and pathophysiology in the light of new advances. Diabetologia. 28:574-578.

25. Samols, E., G. C. Weir, and S. Bonner-Weir. 1986. Intra-islet insulin-glucagon-somatostatin relationships. Clin. Endocrinol. Metab. 15:33-58.

26. Samols, E., and J. I. Stagner. 1988. Intra-islet regulation. Am. J. Med. 85(5A):31-35.

27. Schauder, P., C. McIntosh, J. Arends, R. Arnold, M. Frerichs, and W. Creutzfeld. 1976. Somatostatin and insulin release from iso- lated rat pancreatic islets stimulated by glucose. FEBS (Fed. Eur. Biochem. Soc.) Lett. 68:225-227.

28. Rappaport, E. B., J. B. Young, and L. Landsberg. 1982. Effects of 2-deoxy-D-glucose on the cardiac sympathetic nerves and the adrenal medulla in the rat: Further evidence for a dissociation of sympathetic nervous system and adrenal medullary responses. Endocrinology. 110:650-656.

29. Palmer, J. P., and D. Porte, Jr. 1982. Neural control of glucagon secretion. In Handbook of Experimental Pharmacology. P. J. Lefebvre, editor. Springer-Verlag, Berlin. 115-132.

30. Hisatomi, A., H. Maruyama, L. Orci, M. Vasko, and R. H. Unger. 1985. Adrenergically mediated intrapancreatic control of the glucagon response to glucopenia in the isolated rat pancreas. J. Clin. Invest. 75:420-426.

31. Klaff, L. J., B. E. Dunning, and G. J. Taborsky. 1988. Somatostatin-28 does not regulate islet function in the dog. Endocrinology. 123:2668-2674.

32. Louie, D. S., J. A. Williams, and C. Owyang. 1985. Action of pancreatic polypeptide on rat pancreatic secretion: in vivo and in vitro. Am. J. Physiol. 249:G489-495.

33. Weir, G. C., J. L. Leahy, and S. Bonner-Weir. 1986. Experimental reduction of B-cell mass: Implications for the pathogenesis of diabetes. Diabetes/Metab. Rev. 2:125-161.

34. Hermansen, K., O. Schmitz, and H. Orskov. 1985. Reversal of $D$ - and A-cell insensitivity to glucose in alloxan-diabetic dogs by treatment with the artificial beta cell (Biostator). Diabetes. 34:260-266.

35. Weir, G. C., S. D. Knowlton, R. F. Atkins, K. X. McKennan, and D. B. Martin. 1976. Glucagon secretion from the perfused pancreas of streptozotocin-treated rats. Diabetes. 25:275-282.

36. Asplin, C. M., T. L. Paquette, and J. P. Palmer. 1981. In vivo inhibition of glucagon secretion by paracrine beta cell activity in man. J. Clin. Invest. 68:314-318.

37. Raskin, P., I. Aydin, and R. H. Unger. 1976. Effect of insulin on the exaggerated glucagon response to arginine stimulation in diabetes mellitus. Diabetes. 25:227-229.

38. Fukuda, M., A. Tanaka, Y. Tahara, H. Ikegami, Y. Yamamoto, Y. Kumahara, and K. Shima. 1988. Correlation between minimal secretory capacity of pancreatic beta cells and stability of diabetic control. Diabetes. 37:81-88.

39. Bolli, G. B., E. Tsalikian, M. W. Haymond, P. E. Cryer, and J. E. Gerich. 1984. Defective glucose counterregulation after subcutaneous insulin in non-insulin-dependent diabetes mellitus. J. Clin. Invest. 73:1532-1541, 1984.

40. Hosker, J. P., M. A. Burnett, D. R. Matthews, and R. C. Turner. 1988. Suppression of insulin secretion by falling plasma glucose levels is impaired in type 2 diabetes. Diabetic Med. 5:856-860.

41. Leahy, J. L., and G. C. Weir. 1985. Unresponsiveness to glucose in a streptozotocin model of diabetes. Inappropriate insulin and glucagon responses to a reduction of glucose concentration. Diabetes. 34:653-659. 